\title{
A Characterization of Symmetric Stable Distributions
}

\author{
Wiktor Ejsmont \\ Department of Mathematics and Cybernetics, Wrocław University of Economics, Ulica Komandorska 118/120, 53-345 Wrocław, Poland
}

Correspondence should be addressed to Wiktor Ejsmont; wiktor.ejsmont@ue.wroc.pl

Received 29 December 2015; Revised 21 March 2016; Accepted 27 March 2016

Academic Editor: Gen Qi Xu

Copyright (C) 2016 Wiktor Ejsmont. This is an open access article distributed under the Creative Commons Attribution License, which permits unrestricted use, distribution, and reproduction in any medium, provided the original work is properly cited.

Characterization problems in probability are studied here. Using the characteristic function of an additive convolution we generalize some known characterizations of the normal distribution to stable distributions. More precisely, if a distribution of a linear form depends only on the sum of powers of the certain parameters, then we obtain symmetric stable distributions.

\section{Introduction}

The original motivation for this paper comes from a desire to understand the results about characterization of normal distribution which were shown in [1]. In this paper, the author provides characterizations of the normal distribution using a certain invariance of the noncentral chi-square distribution. More precisely, let statistic $\sum_{i=1}^{n} a_{i} X_{i}+Y+Z$ have a distribution which depends only on $\sum_{i=1}^{n} a_{i}^{2}$, with $a_{i} \in \mathbb{R}$, $1 \leq m<n$, where $\left(X_{1}, \ldots, X_{m}, Y\right)$ and $\left(X_{m+1}, \ldots, X_{n}, Z\right)$ are independent random vectors with all moments, and $X_{i}$ are nondegenerate; then $X_{i}$ are independent and have the same normal distribution with zero means and $\operatorname{cov}\left(X_{i}, Y\right)=$ $\operatorname{cov}\left(X_{i}, Z\right)=0$ for $i \in\{1, \ldots, n\}$. The proof of the above theorem is divided into two parts: first, it is proved that this result holds for two random variables. Second, it is shown using the properties of multidimensional normal distribution. The additional moment assumption is due to the fact that the author uses a method of cumulants. An alternative method of proof (more direct and straightforward one) allows us to weaken some of the technical assumptions used in the above references and generalize it to a symmetric stable distribution. The paper is organized as follows. In Section 2 we review basic facts about characteristic function. Next in Section 3 we state and prove the main results.

\section{A Characteristic Function}

In this paper we denote by $\mu_{X}(d x)$ a probability measure of random variable $X$. If $X$ is a random variable defined on a probability space $(\Omega, \Sigma, P)$, then the expected value of $X$, denoted by $E(X)$, is defined as the Lebesgue integral:

$$
E(X)=\int_{\Omega} X(\omega) \mathbb{P}(d \omega) .
$$

A characteristic function is simply the Fourier transform, in probabilistic language. The characteristic function of a probability measure $\mu$ on $\mathbb{R}$ is the function $\varphi: \mathbb{R} \rightarrow \mathbb{C}$ :

$$
\varphi_{\mu}(t)=\int_{\mathbb{R}} \exp (i t x) d \mu(d x) .
$$

When we speak of the characteristic function $\varphi_{X}$ of a random variable $X$, we have the characteristic function $\varphi_{\mu_{X}}$ of its distribution $\mu_{X}$ in mind. Note, moreover, that

$$
\varphi_{\mu}(t)=E[\exp (\text { it } X)]
$$

Apparently, it is not accidental that the characteristic function encodes the most important information about the associated random variables. The underlying reason may well reside in the following three important properties:

(i) The Gaussian distribution $N(\mu, \sigma)$ has the characteristic function $\varphi(t)=\exp \left(i \mu t-\sigma^{2} t / 2\right)$.

(ii) The symmetric $\alpha$-stable distribution has the characteristic function $\varphi(t)=\exp \left(-c|t|^{\alpha}\right)$, where $\alpha \in(0,2\rangle$ and $c>0$. For the special cases of parameter $\alpha$, we get

(1) the upper bound $\alpha=2$ corresponding to the normal distribution, 
(2) $\alpha=1$ corresponding to the Cauchy distribution,

(3) for $\alpha=0.5$ the distribution reduces to a Lévy distribution.

(iii) Random variables $X_{1}, \ldots, X_{n}$ are independent if and only if for all $a_{1}, \ldots, a_{n} \in \mathbb{R}$ the joint characteristic function (i.e., the linear combination of the $X_{i}$ 's) satisfies

$$
\begin{aligned}
& E \exp \left(i a_{1} X_{1}+\cdots+i a_{n} X_{n}\right) \\
& \quad=E \exp \left(i a_{1} X_{1}\right) \cdots E \exp \left(i a_{n} X_{n}\right) .
\end{aligned}
$$

\section{The Characterization Theorem}

The main result of this paper is the following characterization of the symmetric $\alpha$-stable distribution in terms of independent random vectors.

Theorem 1. Let $X=\left(X_{1}, \ldots, X_{m}\right)$ and $\left(X_{m+1}, \ldots, X_{n}\right)$ be independent random vectors, where the distribution of $X_{i}$ is not Dirac measure and let statistic $\sum_{i=1}^{n} a_{i} X_{i}+Z$ have a distribution which depends only on $\sum_{i=1}^{n}\left|a_{i}\right|^{\alpha}$, for all $a_{1}, \ldots, a_{n} \in \mathbb{R}, 1 \leq$ $m<n, Z$ being an arbitrary random variable, and $\alpha \in(0,2\rangle$. Then $X_{i}$ are independent and have the same symmetric $\alpha$-stable distribution. Additionally, if $Z \in L^{2}(\Omega, \Sigma, P)$ and $\alpha=2$ then $\operatorname{cov}\left(X_{i}, Z\right)=0$ for $i \in\{1, \ldots, n\}$.

Proof. We write $\Theta=r\left(\theta_{1}, \theta_{2}, \ldots, \theta_{n}\right)$, where $\left|\theta_{1}\right|^{\alpha}+\left|\theta_{2}\right|^{\alpha}+\cdots+$ $\left|\theta_{n}\right|^{\alpha}=1$. We focus on characteristic function $\varphi_{\sum_{i=1}^{n} r \theta_{i} X_{i}+Z}(t)$ of $\sum_{i=1}^{n} r \theta_{i} X_{i}+Z$. Then for $r>0$ we have

$$
\begin{aligned}
\varphi_{\sum_{i=1}^{n} r \theta_{i} X_{i}+Z}\left(\frac{t}{r}\right) & =E e^{i\left(\sum_{i=1}^{n} r \theta_{i} X_{i}+Z\right) t / r} \\
& =E e^{i\left(\theta_{1} S_{1}+\theta_{2} S_{2}+\cdots+\theta_{n} X_{n}\right) t+i Z t / r} .
\end{aligned}
$$

Since the hypothesis implies that left side of (5) does not depend on $\Theta / r=\theta=\left(\theta_{1}, \theta_{2}, \ldots, \theta_{n}\right)$, then

$$
\lim _{r \rightarrow+\infty} \varphi_{\sum_{i=1}^{n} r \theta_{i} X_{i}+Z}\left(\frac{t}{r}\right)=E e^{i\left(\theta_{1} X_{1}+\theta_{2} X_{2}+\cdots+\theta_{n} X_{n}\right) t}
$$

does not depend on $\theta$. In particular we have that the distribution of a statistic

$$
\sum_{i=1}^{n} a_{i} X_{i}=\left(\sum_{i=1}^{n}\left|a_{i}\right|^{\alpha}\right)^{1 / \alpha}\left(\sum_{i=1}^{n} \frac{a_{i}}{\left(\sum_{i=1}^{n}\left|a_{i}\right|^{\alpha}\right)^{1 / \alpha}} X_{i}\right)
$$

depends on $\sum_{i=1}^{n}\left|a_{i}\right|^{\alpha}$ only. Let $h\left(\sum_{i=1}^{n}\left|a_{i}\right|^{q}\right)=E e^{i \sum_{i=1}^{n} a_{i} X_{i}}$. Because of the independence of $\left(X_{1}, \ldots, X_{m}\right)$ and $\left(X_{m+1}, \ldots, X_{n}\right)$ we may write

$$
h\left(\sum_{i=1}^{n}\left|a_{i}\right|^{\alpha}\right)=E e^{i \sum_{i=1}^{m} a_{i} X_{i}} E e^{i \sum_{i=m+1}^{n} a_{i} X_{i}} .
$$

Evaluating (8) first when $a_{m+1}=\cdots=a_{n}=0$ and then when $a_{1}=\cdots=a_{m}=0$, we get $h\left(\sum_{i=1}^{m}\left|a_{i}\right|^{\alpha}\right)=E e^{i \sum_{i=1}^{m} a_{i} X_{i}}$ and
$h\left(\sum_{i=m+1}^{n}\left|a_{i}\right|^{\alpha}\right)=E e^{i \sum_{i=m+1}^{n} a_{i} X_{i}}$, respectively. Substituting this into (8), we see

$$
h\left(\sum_{i=1}^{n}\left|a_{i}\right|^{\alpha}\right)=h\left(\sum_{i=1}^{m}\left|a_{i}\right|^{\alpha}\right) h\left(\sum_{i=m+1}^{n}\left|a_{i}\right|^{\alpha}\right) .
$$

Note that $h(u)$ is continuous in $u \in[0, \infty)$, which implies $h(u)=e^{c u}$ (see Aczel [2], page 31), and so we have $h\left(\sum_{i=1}^{n}\left|a_{i}\right|^{\alpha}\right)=e^{c\left(\sum_{i=1}^{n} a_{i}^{\alpha}\right)}$. Thus we have actually proved that $X_{1}, \ldots, X_{n}$ have the same symmetric $\alpha$-stable distribution. But since we know that the distributions of $X_{i}$ are symmetric stable, the independence of random variables $X_{1}, \ldots, X_{n}$ follows from the observation that

$$
\begin{aligned}
h\left(\sum_{i=1}^{n}\left|a_{i}\right|^{\alpha}\right) & =E e^{i \sum_{i=1}^{n} a_{i} X_{i}}=e^{c\left(\sum_{i=1}^{n}\left|a_{i}\right|^{\alpha}\right)} \\
& =E \exp \left(i X_{1} a_{1}\right) \cdots E \exp \left(i X_{n} a_{n}\right) .
\end{aligned}
$$

If $\alpha=2$ and $E\left(Z^{2}\right)<\infty$ then $\operatorname{cov}\left(X_{i}, Z\right)<\infty$ and because of the independence of $X_{1}, \ldots, X_{n}$ we may write

$$
\begin{aligned}
V\left(\sum_{i=1}^{n} a_{i}^{2}\right)= & \operatorname{Var}\left(\sum_{i=1}^{n} a_{i} X_{i}+Z\right) \\
= & 2 \sum_{i=1}^{n} a_{i} \operatorname{cov}\left(X_{i}, Z\right)+\left(\sum_{i=1}^{n} a_{i}^{2}\right) \operatorname{Var}\left(X_{1}\right) \\
& +\operatorname{Var}(Z)
\end{aligned}
$$

because $\operatorname{cov}\left(X_{i}, X_{j}\right)=0$ for $i \neq j$ and $\operatorname{Var}\left(X_{1}\right)=$ $\cdots=\operatorname{Var}\left(X_{n}\right)$. This implies that linear combination $\sum_{i=1}^{n} a_{i} \operatorname{cov}\left(X_{i}, Z\right)$ is constant on sphere $\left\{\left(a_{1}, \ldots, a_{n}\right)\right.$ : $\left.\sum_{i=1}^{n} a_{i}^{2}=1\right\}$ which gives $\operatorname{cov}\left(X_{i}, Z\right)=0$.

The above consideration gives us the following results of Ejsmont [1] and Cook [3], respectively.

Corollary 2 (the main result of Ejsmont [1]). Let $\left(X_{1}, \ldots\right.$, $\left.X_{m}, Y\right)$ and $\left(X_{m+1}, \ldots, X_{n}, Z\right)$ be independent random vectors with all moments, where $X_{i}$ are nondegenerate, and let statistic $\sum_{i=1}^{n} a_{i} X_{i}+Y+Z$ have a distribution which depends only on $\sum_{i=1}^{n} a_{i}^{2}$ for all $a_{i} \in \mathbb{R}$ and $1 \leq m<n$. Then $X_{i}$ are independent and have the same normal distribution with zero means and $\operatorname{cov}\left(X_{i}, Y\right)=\operatorname{cov}\left(X_{i}, Z\right)=0$ for $i \in\{1, \ldots, n\}$.

Corollary 3 (the main result of Cook [3]). Let $\left(X_{1}, \ldots, X_{m}\right)$ and $\left(X_{m+1}, \ldots, X_{n}\right)$ be independent random vectors, where $X_{i}$ are nondegenerate, and let statistic $\sum_{i=1}^{n}\left(X_{i}+a_{i}\right)^{2}$ have a distribution which depends only on $\sum_{i=1}^{n} a_{i}^{2}, a_{i} \in \mathbb{R}$ and $1 \leq m<n$. Then $X_{i}$ are independent and have the same normal distribution with zero means.

Proof. If we put $Z=\sum_{i=1}^{n} X_{i}^{2}$ and $\alpha=2$ in Theorem 1 then we get

$$
\sum_{i=1}^{m} a_{i} X_{i}+\sum_{i=m+1}^{n} a_{i} X_{i}+Z=\sum_{i=1}^{n}\left(X_{i}+\frac{a_{i}}{2}\right)^{2}-\frac{1}{4} \times \sum_{i=1}^{n} a_{i}^{2} .
$$


This means that the distribution of $\sum_{i=1}^{m} a_{i} X_{i}+\sum_{i=m+1}^{n} a_{i} X_{i}+$ $Z$ depends only on $\sum_{i=1}^{n} a_{i}^{2}$, which by Theorem 1 implies the statement.

Here we state the Herschel-Maxwell theorem in modern notation (see, e.g., [4] or [5]). This theorem can be also obtained from Theorem 1 by considering $m=1$ and $n=2$ as well as $Z=0$ and $\alpha=2$ (the proof is left to the reader).

Theorem 4. Let $X, Y$ be independent random variables and $a_{1}, a_{2}$ real numbers such that $a_{1}^{2}+a_{2}^{2}=1$. Then $X, Y$ are normally distributed with zero means if and only if $a_{1} X+a_{2} Y$ is distributed identically as $X$ for any $\left(a_{1}, a_{2}\right) \in \mathbb{R}^{2}$.

Open Problem. Kagan and Letac [6] formulate the following theorem: Let $n$ be a fixed integer $n \geq 3$. Let $X_{1}, X_{2}, \ldots, X_{n}$ be independent identically distributed random variables. In the Euclidean space $\mathbb{R}^{n}$ consider the linear subspace $E=1^{\perp}$, that is, the set $\left\{\left(a_{1}, a_{2}, \ldots, a_{n}\right): a_{1}+a_{2}+\cdots+a_{n}=0\right\}$. Then the following characterizations hold: If for all $a \in E$ the distribution of the random variable

$$
\sum_{i=1}^{n}\left(X_{i}-\bar{X}+a_{i}\right)^{2}
$$

depends only on $\|a\|^{2}=a_{1}^{2}+a_{2}^{2}+\cdots+a_{n}^{2}$, then $X_{i}$ 's are normally distributed.

A key role in the proof of these results is played by Marcinkiewicz' theorem: if $Q(x)$ is a polynomial and $\exp (Q(x))$ is the characteristic function of some probability distribution, then the degree of $Q$ is less than or equal to two. Finally, we present the conjecture (Theorem 1 cannot be applied here).

Conjecture 5. Let $\left(X_{1}, \ldots, X_{m}\right)$ and $\left(X_{m+1}, \ldots, X_{n}\right)$ be independent random vectors, where $X_{i}$ are nondegenerate, and the distribution of the random variable

$$
\sum_{i=1}^{n}\left(X_{i}-\bar{X}+a_{i}\right)^{2}
$$

depends only on $\|a\|^{2}=a_{1}^{2}+a_{2}^{2}+\cdots+a_{n}^{2}$, where $a=$ $\left(a_{1}, \ldots, a_{n}\right) \in E$, and then $X_{i}$ 's are normally distributed independent random variables.

\section{Competing Interests}

The author declares having no competing interests.

\section{Acknowledgments}

The author would like to thank M. Bożejko for several discussions and helpful comments during the preparation of this paper. The work was partially supported by the Narodowe Centrum Nauki, Grant no. 2014/15/B/ST1/00064, and by the Austrian Science Fund (FWF) Project no. P 25510-N26.

\section{References}

[1] W. Ejsmont, "A characterization of the normal distribution by the independence of a pair of random vectors," Statistics and Probability Letters, vol. 114, pp. 1-5, 2016.

[2] J. Aczel, Lectures on Functional Equations and Their Applications, Academic Press, New York, NY, USA, 1966.

[3] L. Cook, "A characterization of the normal distribution by the independence of a pair of random vectors and a property of the noncentral chi-square statistic," Journal of Multivariate Analysis, vol. 1, pp. 457-460, 1971.

[4] W. Bryc, The Normal Distribution: Characterizations with Applications, vol. 100 of Lecture Notes in Statistics, Springer, New York, NY, USA, 1995.

[5] A. M. Kagan, Y. V. Linnik, and C. R. Rao, Characterization Problems in Mathematical Statistics, John Wiley \& Sons, New York, NY, USA, 1973.

[6] A. M. Kagan and G. Letac, "Characterization of the normal distribution through the power of a one-way ANOVA," Journal of Statistical Planning and Inference, vol. 77, no. 1, pp. 1-9, 1999. 


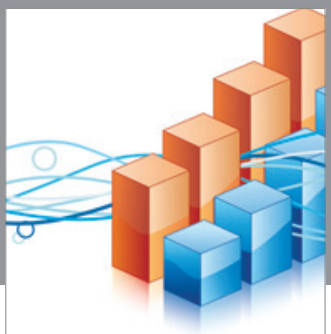

Advances in

Operations Research

vatem alat4

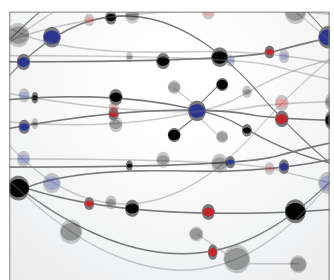

\section{The Scientific} World Journal
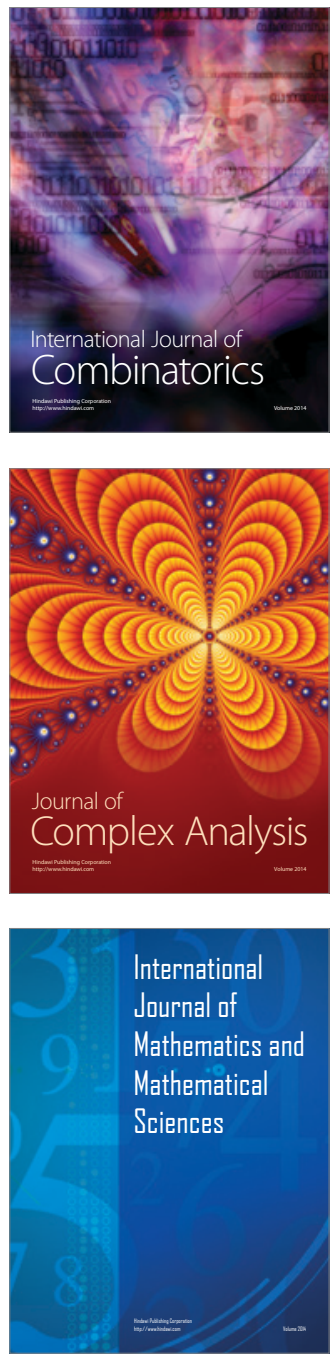
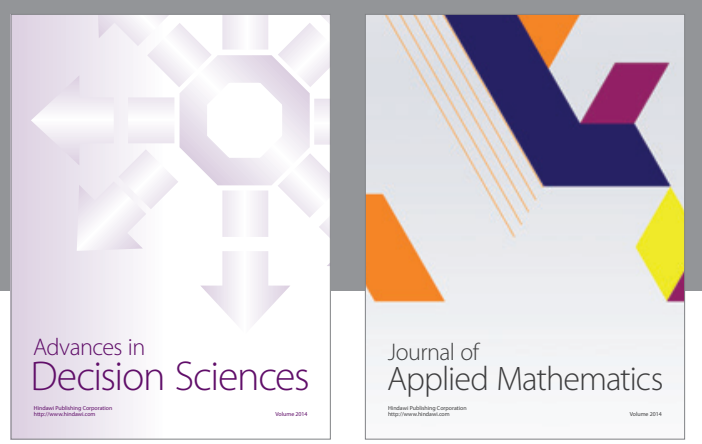

Algebra

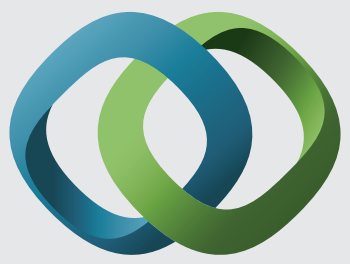

\section{Hindawi}

Submit your manuscripts at

http://www.hindawi.com
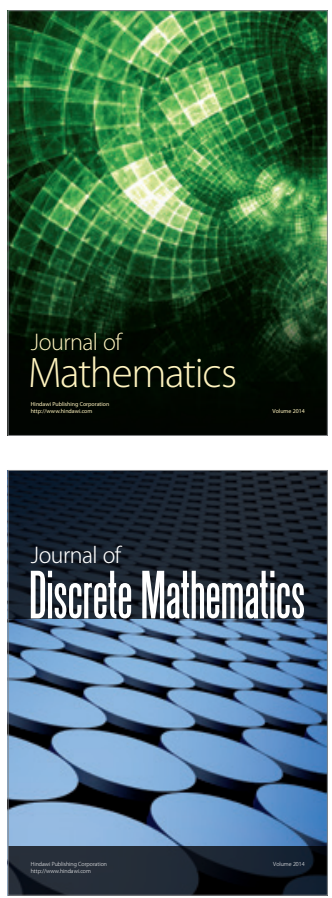

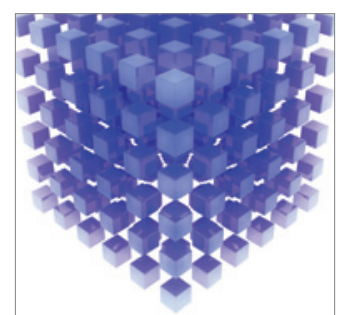

Mathematical Problems in Engineering
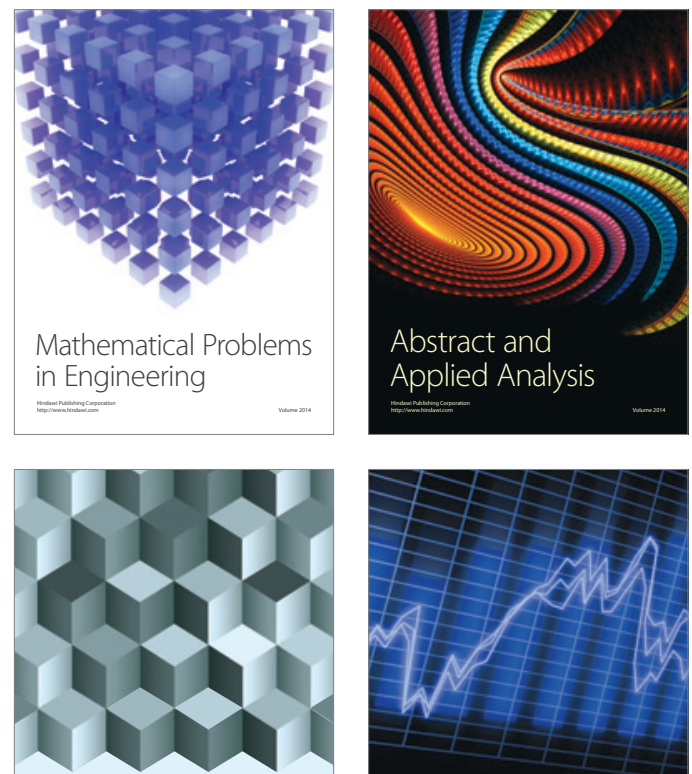

Journal of

Function Spaces

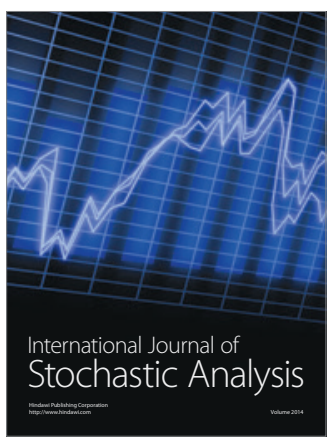

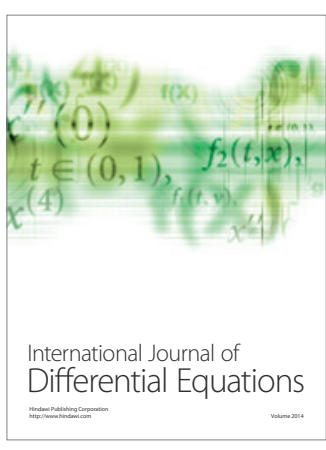
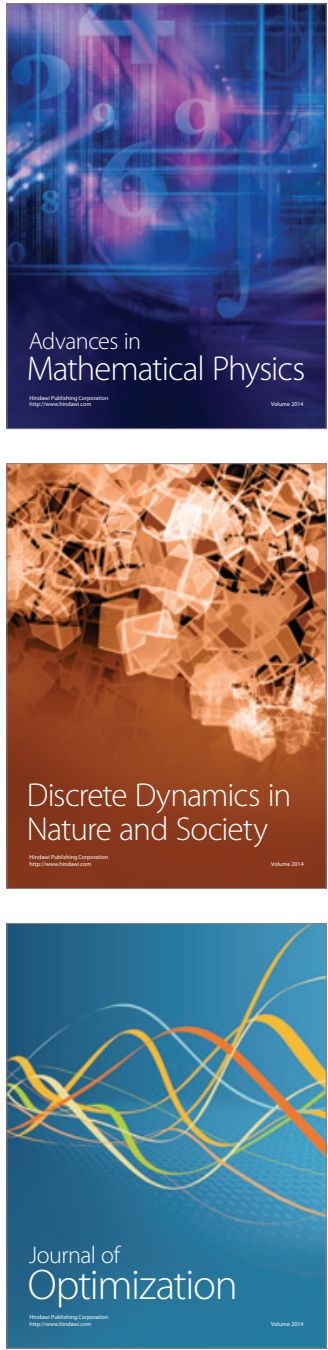